\title{
New targets and developments in lipoproteins control
}

This article was published in the following Dove Press journal:

Research Reports in Clinical Cardiology

24 May 2013

Number of times this article has been viewed

\begin{abstract}
Giuseppe Danilo Norata ${ }^{1-3}$
'Department of Pharmacological and Biomolecular Sciences, Università degli Studi di Milano, Italy; ${ }^{2}$ Center for the Study of Atherosclerosis, Società Italiana Studio Aterosclerosi, Ospedale Bassini, Cinisello Balsamo, Italy; ${ }^{3}$ The Blizard Institute, Centre for Diabetes, Barts and The London School of Medicine and Dentistry, Queen Mary University, London, UK
\end{abstract}

Correspondence: Giuseppe Danilo Norata

Department of Pharmacological and Biomolecular Sciences, Università degli Studi di Milano, via Balzaretti 9 20I33, Milan, Italy Email danilo.norata@unimi.it

\begin{abstract}
Statins provide a very effective approach in reducing plasma cholesterol levels and cardiovascular risk. However, the proportion of patients who fail to achieve desirable plasma lipid levels ranges from $16 \%-53 \%$, worldwide. This percentage reaches up to $80 \%$ in patients with familial hypercholesterolemia. Additionally, many patients are unable to tolerate statins, particularly at the highest approved dose level. New treatments that aggressively reduce lipid levels in patients with severe hypercholesterolemia, or those unable to reach their lipid targets, are therefore required. The most promising approaches in this context, such as inhibitors of the synthesis of apolipoprotein B (apoB) containing lipoproteins (apoB silencing or microsomal triglyceride transfer protein [MTP] inhibition) or proprotein convertase subtilisin/kexin type 9 (PCSK9) blockers, all decrease low-density lipoprotein (LDL) extensively. Increasing low levels of high-density lipoprotein (HDL) cholesterol via cholesteryl ester transfer protein inhibitors or apolipoprotein A-1 (ApoA-1) inducers and improving their quality with HDL or ApoA-1 mimetics represent also important options. Drugs affecting HDL, however, may not be all alike and require adequate scrutiny of the mechanisms involved. Until we have a better understanding of these issues, further LDL lowering in high-risk patients represents the soundest approach.
\end{abstract}

Keywords: apolipoproteins, lipids, lipoprotein classes, hypercholesterolemia, synthesis, LDL lowering

\section{Introduction}

The discovery of statins provided a very effective approach in reducing cardiovascular risk, as documented by the results obtained in clinical trials and in clinical practice. This class of drugs inhibits 3-hydroxy-3-methylglutaryl-coenzyme A (HMG-CoA) reductase activity, thus halting the conversion of acetyl coenzyme $\mathrm{A}$ and acetoacetyl coenzyme A to mevalonate, a key step in cholesterol synthesis. This inhibition leads to a reduced synthesis of cholesterol in the liver and in an increased expression of hepatic low-density lipoprotein receptor (LDL-R). The latter is responsible for increased LDL cholesterol (LDL-C) and reduced plasma levels of LDL-C and other apolipoprotein (apo)B-containing lipoproteins including microsomal triglyceride-rich particles.

Despite the efficacy and the widespread availability of statins in reducing LDL-C and cardiovascular risk; the proportion of patients who fail to achieve their lipid targets ranges from $16 \%$ to $53 \%$, worldwide. Additionally, many patients are unable to tolerate statins, particularly at the highest approved dose level. Finally, up to $\sim 80 \%$ of patients with severe forms of this disorder, such as familial hypercholesterolemia (FH), are unable to achieve their target levels using conventional therapies, such as 
statins. Although this gap is partially filled when statins are combined to other agents, such as ezetimibe, new treatments that aggressively reduce lipid levels in patients with severe hypercholesterolemia, or those unable to reach their lipid targets, are still required.

In addition, experimental data clearly suggest that other lipoprotein classes beyond LDL play important roles in determining cardiovascular risk. For these reasons, a number of new drugs are under development in this area. The aim of this review is to discuss the available and the future pharmacological strategies for the management of dyslipidemia, including low levels of high-density lipoprotein (HDL) cholesterol. Emerging approaches aimed at reducing elevated levels of plasma triglycerides and lipoprotein(a) were recently presented elsewhere ${ }^{1,2}$ and will not be discussed here.

\section{Current developments for lowering LDL Interfering with lipoprotein synthesis: apoB silencing, MTP inhibitors}

Hepatic biosynthesis of very low-density lipoprotein (VLDL) is dependent on two dominant proteins, namely apolipoprotein B (apoB) and microsomal triglyceride (TG) transfer protein (MTP). ApoB is an obligatory structural component of VLDL and requires progressive lipidation, mediated by the resident endoplasmic reticulum chaperone MTP, to maintain conformational integrity and folding during the process of lipoprotein assembly. Interfering with this process is therefore an attractive approach for reducing lipoprotein synthesis and decreasing plasma VLDL-C and LDL-C concentration. The possibility of targeting apoB during the process of gene translation is under extensive investigation. One approach to block messenger ribonucleic acid (mRNA) translation of a gene is by using a single-strand antisense oligonucleotide (ASO) that is complementary to the mRNA. Following hybridization to the mRNA, the ASO inhibits translation and splicing and leads to degradation of the mRNA by ribonuclease (RNase). ${ }^{3}$ ASO kinetics are characterized by a large and rapid distribution to the liver following parenteral administration, thus making this approach quite attractive for inhibiting mRNA posttranscriptional processing. ${ }^{1,2}$ This results in a reduced synthesis of proteins in the liver, such as apoB ${ }^{4}$ (Figure 1). Preclinical studies demonstrated that ASOs targeting apoB are quite effective in mice in reducing apoB mRNA liver levels in a dose-response manner, ${ }^{5}$ followed by a reduction in circulating LDL-C concentration, LDL particle number, circulating TG, and lipoprotein(a) (Lp[a]), while chylomicrons, which contain apoB-48, were spared, because of the high distribution of ASOs to the liver.

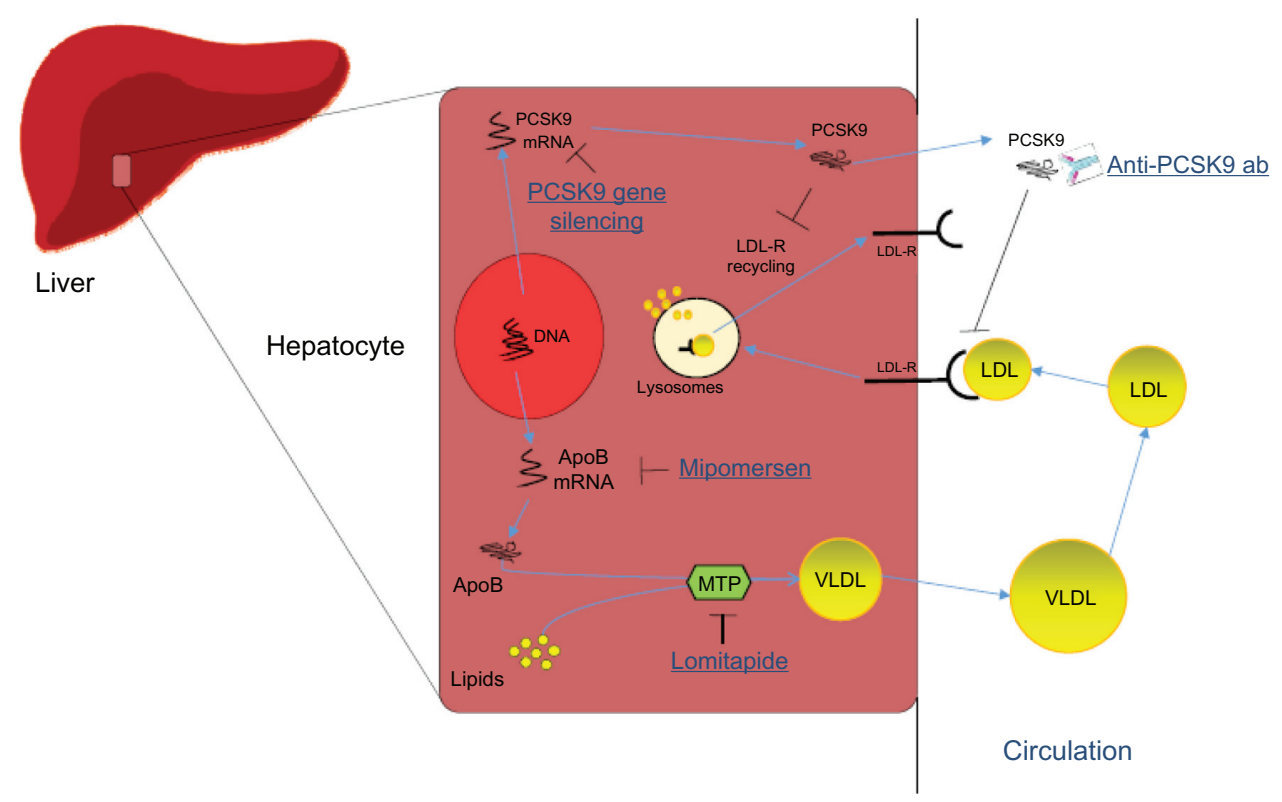

Figure I Emerging targets and agents for LDL-cholesterol lowering.

Notes: Emerging therapeutic agents for LDL-C lowering have been designed: (a) to reduce apoB containing lipoprotein synthesis in the liver; or (b) to improve LDL catabolism. Mipomersen silences apoB expression while lomitapide inhibits MTP activity. Blocking PCSK9 activity by monoclonal antibodies, or through gene silencing promotes LDL-R activity.

Abbreviations: LDL, low-density lipoprotein; LDL-C, low-density lipoprotein catabolism; apoB, apolipoprotein B; MTP, microsomal triglyceride transfer protein; PCSK9, proprotein convertase subtilisin/kexin type 9; mRNA, messenger RNA; LDL-R, LDL-receptor; VLDL, very low-density lipoprotein; ab, antibody. 
Mipomersen is an ASO-targeting apoB which leads to a dose-dependent reduction in apoB and total cholesterol. ${ }^{6}$ This approach was effective in Phase II and Phase III clinical studies, in combination with statin therapy with or without ezetimibe, bile acid sequestrant, and/or niacin. The treatment was effective also in patients with $\mathrm{FH},{ }^{6}$ as well as in monotherapy in individuals with mild to moderate hyperlipidemia ${ }^{7}$ and in high-risk statin-intolerant patients. ${ }^{8}$ Overall, mipomersen provided significant further reduction in LDL-C $(\sim 30 \%)$ and other lipids when added to conventional lipid therapy. The most common adverse effects were injection-site reactions and flu-like symptoms. Liver fat accumulation was also observed in both Phase II and Phase III studies and is in line with the mechanism of action of the drug. ${ }^{2}$ As of January 2013, mipomersen has been approved by the US Food and Drug Administration (FDA) for use in homozygous FH with a warning for the possible clinical consequences of the liver fat accumulation (http:// www.fda.gov/NewsEvents/Newsroom/PressAnnouncements/ ucm337195.htm). The European Medicines Agency Committee for Medicinal Products for Human Use, however, issued a negative opinion on mipomersen based on safety issues, and further assessment is awaited.

MTP, found in the endoplasmic reticulum of hepatocytes and enterocytes, mediates the formation of apoB-containing lipoproteins in the liver and in the intestine. ${ }^{9}$ Mutations in the gene encoding MTP can cause abetalipoproteinemia, a rare genetic disease characterized by an absence of apoB-containing lipoproteins and severe malabsorption of fat and fat-soluble vitamins. ${ }^{9}$ The genetic defect underlying abetalipoproteinemia suggests that inhibiting MTP may reduce circulating concentrations of cholesterol and apoB-containing lipoproteins (Figure 1). The MTP inhibitor lomitapide is currently in Phase III testing. The drug, tested in monotherapy or in combination with conventional lipid-lowering therapy in homozygous $\mathrm{FH}^{10}$ or in patients with hypercholesterolemia (LDL-C $130 \mathrm{mg} /$ $\mathrm{dL}-250 \mathrm{mg} / \mathrm{dL}),{ }^{11}$ showed a reduction in circulating LDL$\mathrm{C}$, apoB, total cholesterol, non-high-density lipoprotein cholesterol (non-HDL-C) and Lp(a) levels. Steatorrhea related to lomitapide treatment was effectively reduced by a fat-restricted diet; adverse effects, such as elevated liver enzymes and hepatic fat accumulation (expected from the mechanism of action), were reported and may restrict the patient population for this drug. However, for patients with homozygous FH that cannot be controlled with conventional lipid-lowering therapy, MTP inhibition may be a beneficial approach.
In December 2012, lomitapide was approved by the US FDA for the treatment of patients with homozygous FH. To date, no cases of suspected drug-induced liver injury have been observed in lomitapide-treated subjects. As the liver lipid accumulation may vary greatly from patient to patient, this matter requires careful consideration and longer term follow-up to exclude specific changes leading to fibrosis and cirrhosis, especially in the light of the very low-fat diet that the FH patients were adhering to and the low-fat liver content at baseline.

\section{Promoting LDL-receptor activity: PCSK9 inhibitors}

Cholesterol homeostasis is regulated by the LDL-R through its binding and uptake of circulating apoB-containing lipoproteins which are then internalized into the liver cell. The key mechanism associated with statins' action involves the increase of LDL-R expression on the hepatocyte surface, followed by increased LDL turnover and reduction of plasma cholesterol levels. This mechanism is partially dampened by a negative feedback response associated with the induction of the expression and secretion of proprotein convertase subtilisin/kexin type 9 (PCSK9), ${ }^{12}$ a serine protease which promotes the degradation of LDL-R, ${ }^{13}$ thus attenuating, at least in part, the lipid-lowering efficacy of statins and ezetimibe. ${ }^{14}$ More recently it was suggested that, in hepatocytes, PCSK9 directly interacts with apoB and reduces its intracellular degradation, resulting in increased apoB output independently of the LDL-R. ${ }^{15}$ The PCSK9/apoB interaction inhibits intracellular degradation of apoB via the autophagosome/lysosome pathway, and this, in turn, results in increased secretion of apoB-containing lipoproteins and increased levels of cholesterol and triacylglycerol (TAG). ${ }^{15}$

Given that PCSK9 acts both intracellularly, as a chaperone directing the LDL-R to the lysosomes or promoting apoB secretion, and in the circulation, by promoting LDL-R internalization, ${ }^{14}$ the possibility of inhibiting PCSK9 represents a logical step to enhance the lipid-lowering effect of conventional agents ${ }^{14}$ (Figure 1). To this end, at least five different human monoclonal antibodies and three gene-silencing approaches are under development. Among a series of antibodies against PCSK9, clinical trial results are available for two of them, SAR236553/ REGN $727^{16}$ and AMG 145, ${ }^{17}$ and these compounds are both in Phase II or Phase III development. A number of additional anti-PCSK9 monoclonal antibodies, in earlier clinical development, are currently being investigated for 
potential use in humans, including 1B20, PF-04950615/ RN-316, and LGT 209. ${ }^{2}$

To date the largest body of information is available for REGN727/SAR236553, a fully human monoclonal antibody, which binds to the catalytic domain of PCSK9 that interacts with LDL-R. Overall, results from Phase I and Phase II clinical trials suggest that subcutaneous injections of SAR236553/REGN727 dose dependently reduce PCSK9 activity and produce significant additional reductions in LDL-C, as well as in non-HDL-C independently of statin treatment. The antibody was generally well-tolerated over the treatment period, with no drug-related adverse effects on liver function tests or other laboratory parameters, and no serious treatment-emergent adverse effects. ${ }^{16,18,19}$ The number of injection-site reactions (including erythema, pruritus, swelling, hematoma, and rash) was generally low, and the few reported were mild in severity. ${ }^{18}$

AMG 145 is another fully human monoclonal antibody which also binds specifically to human PCSK9. Phase I data in subjects on stable statin therapy demonstrated a dosedependent decrease in LDL-C and unbound PCSK9 with increasing subcutaneous doses of AMG 145. LDL-C was lowered by up to $81 \%$ at maximal doses, over and above the LDL lowering achieved with statin alone. ${ }^{17}$ Phase I data ${ }^{17}$ indicated no serious adverse events in the AMG 145 group, compared with placebo, no discontinuations from the studies related to adverse events, and only one case of transaminase elevation $>3 \times$ the upper limit of normal. Although the safety results for PCSK9 monoclonal antibodies are encouraging, it should be noted that the trials to date have been relatively short in duration and were conducted in relatively small patient populations. Further trials are therefore required to test the long-term safety and efficacy of PCSK9 monoclonal antibodies in larger and more varied patient populations. In this context, given that statin treatment increases PCSK9 levels, it should be considered that the frequency of injection may be increased accordingly in statin-treated patients for optimal PCSK9 inhibition.

PCSK9 can also be suppressed through gene silencing with the nucleic acid-based therapies. ${ }^{1}$ Among them, the development of SPC5001, a locked nucleic acid-based inhibitor, and that of BMS-844421, an antisense RNA therapy, were terminated during Phase I clinical trials. ALNPCS02, an RNA interference molecule, is being tested in an ongoing Phase I study in healthy volunteers to evaluate safety and tolerability of various doses. In interim data on 20 subjects, robust target protein knockdown was observed at the highest dose tested, with a mean $60 \%$ reduction in plasma PCSK9 levels 3-5 days after administration. In line with PCSK9 genetics, this type of knockdown entailed a mean 39\% reduction in LDL-C, with no drug-related discontinuations or liver enzyme elevations. ${ }^{19}$

A new class of agents, the cholesteryl ester transfer protein (CETP) inhibitors (see the Emerging approaches for increasing HDL paragraph), which were developed on the premise that they would increase HDL-C more than any therapy currently available, are currently emerging as agents able to relevantly reduce LDL-C. Anacetrapib at $100 \mathrm{mg}$ /day in patients with coronary heart disease or risk equivalent conditions (peripheral artery disease, cerebrovascular disease, diabetes, or a 10-year Framingham Risk Score $>20 \%$ ) reduced LDL-C by $40 \%$ with no changes in blood pressure or serum aldosterone levels. ${ }^{21,22}$ In a Phase II study, evacetrapib (30 mg, $100 \mathrm{mg}$, or $500 \mathrm{mg}$ daily) increased HDL-C levels (+53.6\% to $+128.8 \%)$ and reduced LDL-C $(-13.6 \%$ to $-35.9 \%)$ compared to placebo. Evacetrapib $100 \mathrm{mg}$ daily in combination with statins increased HDL-C levels by $80 \%$ and decreased LDL-C levels by $12 \%$ above statin monotherapy. ${ }^{23}$ The possibility that, for CETP inhibitors, the benefit may still be largely due to the incremental lowering of LDL-C observed with the more potent inhibitors, should be considered for the transfer of these drugs in the clinical practice.

\section{Emerging approaches for increasing HDL Increasing plasma HDL-C levels: CETP inhibitors, apoA-I inducers}

HDLs possess several physiological activities that may explain their antiatherosclerotic properties. Among them, the most relevant is the ability of HDL to promote the efflux of excess cholesterol from peripheral tissues to the liver for excretion. ${ }^{24,25}$ Is this context, reverse cholesterol transport from macrophages in the atherosclerotic lesions was suggested to influence atherosclerosis by limiting foam cells formation. ${ }^{24,25}$ Of note, the HDL also traffics cholesterol to the gut (the transintestinal cholesterol efflux), adipocytes, and steroidogenic tissues and could also exchange cholesterol esters with apoB containing lipoproteins. Furthermore, apolipoproteins, lipids, and enzymes carried by HDL may perform additional antiatherosclerotic activities. ${ }^{26-30}$

In recent years, the metabolic pathways associated with HDL have been extensively investigated and elucidated, allowing the design of drugs able to interfere with HDL 
catabolism, improve the expression of the main protein constituent, namely apoA-I, or mimic their activity.

The pharmacological approaches under development can be grouped in two major clusters - molecules increasing plasma HDL levels and molecules improving HDL function. It is expected that an increase in HDL levels can be beneficial when associated with an improvement in HDL function.

Recently, a Mendelian randomization analysis revealed that a single nucleotide polymorphism in the endothelial lipase gene (LIPG Asn396Ser) associated with increased HDL-C levels in the population did not decrease the risk of myocardial infarction, despite a $13 \%$ reduction expected from the increased HDL-C levels. ${ }^{31}$ Similarly, a genetic score combining 14 variants exclusively related to HDL-C was not associated with myocardial infarction risk, ${ }^{31}$ further challenging the concept that higher HDL-C levels will automatically translate into lower cardiovascular risk. In spite of these observations, clinical trials are still ongoing with drugs affecting HDL levels.

Cholesteryl ester transfer protein (CETP) is an enzyme involved in the transfer of cholesteryl esters from HDL to LDL and VLDL. This process results in a reduction and remodeling of HDL particles and in an increase of LDL and VLDL levels. Furthermore, CETP transfers TG from VLDL or LDL to HDL, resulting in the formation of TG-enriched HDL, which is easily hydrolyzed by hepatic lipase, leading to TG-rich small HDL that are cleared more rapidly from the circulation. ${ }^{32}$ Under pathological conditions, including atherosclerosis, CETP activity is increased; moreover, in humans, CETP deficiency results in increased HDL levels. Altogether, these observations led to the concept that CETP inhibition is a powerful tool to increase HDL-C, decrease LDL-C and VLDL-C, and reduce the development of atherosclerosis. ${ }^{33}$

The first CETP inhibitor developed, torcetrapib, despite a $72 \%$ increase in HDL-C levels, was withdrawn because of an increased risk of cardiovascular events and death from any cause in the Investigation of Lipid Level Management to Understand its Impact in Atherosclerotic Events (ILLUMINATE) trial. ${ }^{34}$ Retrospectively, this effect was attributed to an off-target effect of torcetrapib, such as the rising of systolic blood pressure by an average $5.4 \mathrm{mmHg},{ }^{35}$ an effect associated with the stimulation of aldosterone synthesis, via pathways independent of CETP inhibition. ${ }^{34,36}$ The possibility that CETP inhibition per se could generate larger cholesterol-enriched HDL with impaired cholesterol efflux potential was also proposed. ${ }^{35}$ However, this was not confirmed by in vitro studies. Among the three newer compounds, dalcetrapib, anacetrapib, and evacetrapib, with different potency toward CETP inhibition (evacetrapib $>$ anacetrapib $>$ dalcetrapib) and apparently lacking the off-target effects of torcetrapib, two remain under development, while that of dalcetrapib was recently halted.

The decision to stop dalcetrapib was based on the dal-OUTCOMES trial interim analysis, which showed that dalcetrapib, in acute coronary syndrome patients, failed to demonstrate a significant reduction in cardiovascular adverse events. ${ }^{37}$ In contrast to the earlier CETP inhibitor, torcetrapib, no safety concerns were reported. In addition, the dal-VESSEL study showed that dalcetrapib reduced CETP activity and increased HDL-C levels without affecting nitric oxide-dependent endothelial function, blood pressure, or markers of inflammation and oxidative stress, ${ }^{38}$ while the dal-PLAQUE study demonstrated some beneficial vascular effects of the drug, including the reduction in total vessel enlargement over 24 months. ${ }^{39}$

While disappointing, the pursuit of an extensive program of clinical trials and basic research to develop dalcetrapib has provided new information on the biology of HDL in both man and animal models, and on CETP inhibition as a viable therapeutic target for raising levels of HDL-C. Several other CETP inhibitors that raise HDL-C levels to a greater extent than dalcetrapib and also significantly lower LDL-C and other novel HDL-raising agents remain under development. Ultimately, the benefits of each of these novel CETP inhibitors must be determined through prospective, randomized, clinical outcome trials.

The life cycle of HDL starts from lipid-poor apoA-I, termed "nascent" or pre $\beta$ - HDL (the latter based on the characteristic electrophoretic mobility), which promotes cholesterol mobilization from the cell membrane mainly through the activation of ATP-binding cassette subfamily A member 1 protein (ABCA-1). Pre $\beta$-HDL accumulates effluxed cholesterol and matures to $\alpha$-HDL, which further promotes cholesterol efflux via the activation of different transporters, including ABCG-1 and scavenger receptor class B member 1 (SR-BI) (Figure 1). Induction of the expression of apoA-I is, therefore, a cornerstone mechanism of drugs so far used to increase HDL levels, including fibrates and nicotinic acid. ${ }^{40}$ A novel, small synthetic molecule, RVX-208, is able to induce apoA-I synthesis and is under development. Preclinical studies in nonhuman primates showed the ability of this compound to increase plasma apoA-I and HDL-C levels by up to $60 \%$ and $97 \%$, respectively. In humans, Asymptomatic atrial fibrillation and Stroke Evaluation in pacemaker patients and the atrial fibrillation Reduction atrial pacing Trial (the 
ASSERT study) showed that the administration of RVX-208 at a dose of $50 \mathrm{mg}, 100 \mathrm{mg}$, or $150 \mathrm{mg}$ twice daily for 12 weeks resulted in increases in apoA-I (up to 5.6\%), HDL-C (by $3.2 \%$ to $8.3 \%$ ), and large HDL particles (by $11.1 \%$ to $21.1 \%$ ), although the primary endpoint of individual pairwise comparisons of apoA-I changes with placebo was not statistically significant. ${ }^{41}$ These findings, although not at the level expected, require further evaluation, perhaps through the investigation of HDL functionality. Furthermore, a clear demonstration that therapies aimed at increasing HDL levels are associated with reduced atherosclerotic events is warranted.

\section{Improving HDL activity}

The rationale behind the development of HDL mimetics is the possibility of mimicking the first phase of the HDL life cycle and promoting cholesterol efflux, mainly from cholesterolloaded cells in the vascular wall, such as macrophages and foam cells.

To this aim, lipid-poor apoA-I-phospholipid complexes have been extensively studied in preclinical models and preliminary studies in humans. So far, different approaches are under investigation. CSL-111 is a complex of native apoA-I and phosphatidylcholine isolated from soybeans which induced a significant reduction in atheroma volume compared with baseline. ${ }^{42}$ The same study showed significantly reduced progression of coronary atherosclerosis in the CSL-111-treated group compared to the placebo. Treatment with CSL-111, however, induced reversible alanine aminotransferase elevations exceeding ten times the normal upper limit in one-third of patients receiving $80 \mathrm{mg}$ of the compound, while no changes were observed in patients receiving $40 \mathrm{mg} / \mathrm{kg}$. ${ }^{42}$ To overcome this limitation, a reformulated version, CSL-112, with greater cholesterol efflux capacity and less hepatotoxicity, is in Phase I study.

A similar approach was tested also by incorporating recombinant apoA-I Milano, which differs from normal apoA-I by a cysteine-to-arginine substitution at amino acid 173. ETC-216 is a complex of apoA-I Milano with phospholipid and in a small clinical study significantly reduced total atheroma volume, measured by intravascular ultrasound, in patients with acute coronary syndrome. ${ }^{43}$ Since 2003, ETC-216 development was halted and, only recently, a different company bought the license and renamed the molecule "MDCO-216," with the aim of starting larger clinical trials soon.

CER-001, a synthetic recombinant human apoA-I HDL mimetic, is being tested in approximately 500 patients
Table I New targets and agents in cholesterol control

\begin{tabular}{lll}
\hline Target & $\begin{array}{l}\text { Pharmacological } \\
\text { approach }\end{array}$ & Drug \\
\hline $\begin{array}{l}\text { LDL lowering } \\
\text { Apolipoprotein B }\end{array}$ & $\begin{array}{l}\text { Gene silencing } \\
\text { Inhibitors }\end{array}$ & $\begin{array}{l}\text { Mipomersen } \\
\text { Lomitapide }\end{array}$ \\
MTP & Monoclonal & SAR236553/REGN727 \\
PCSK9 & Antibodies & AMG I45 \\
HDL increase & Inhibitors & Anacetrapib \\
CETP & & Evacetrapib \\
ApoA-I & Inducers & RVX-208 \\
ApoA-I/HDL & Mimetics & CSL-III \\
& & ETC-216 \\
& & CER-00I \\
& & D-4F
\end{tabular}

Abbreviations: MTP, microsomal triglyceride transfer protein; PCSK9, proprotein convertase subtilisin/kexin type 9; CETP, cholesteryl ester transfer protein; ApoA-I, apolipoprotein A-I; HDL, high-density lipoprotein.

with acute coronary syndrome to determine the effect on atherosclerotic plaque progression/regression as assessed by intravascular ultrasound (Chi square). ${ }^{44}$ Several other complexes of apoA-I and different phospholipids are in preclinical development and will soon enter clinical testing phases. Also, micro RNA were proposed to influence the expression of key genes involved in the HDL life cycle and cholesterol efflux. ${ }^{45}$ Micro RNA-33 represents an interesting target, but further studies are required.

A second approach to improve HDL function is represented by small peptides designed to mimic apoA-I function. The most well-studied of these peptides is $4 \mathrm{~F}$, consisting of 18 amino acids, which was designed to have the lipid-binding properties of apoA-I through a common secondary structure, the class A amphipathic helix. The use of D-amino acids (D-4F) enables oral delivery of this compound by conferring resistance to gastrointestinal proteolytic enzymes. Several preclinical studies showed that $4 \mathrm{~F}$ promotes cholesterol efflux via ABCA1 and SR-BI and possesses anti-inflammatory, antithrombotic, and antioxidant properties. The only available human study of D-4F showed that HDL isolated from subjects treated with a single $300 \mathrm{mg}$ or $500 \mathrm{mg}$ dose of unformulated D-4F had increased inhibition of LDL-induced monocyte chemotaxis, compared to HDL isolated from control subjects. Data on the safety profile of D-4F in humans are not available yet. Overall at least 22 apoA-I mimetics are under development; ${ }^{46}$ however, with the exception of D-4F, the other peptides require parenteral administration and, in humans, data on efficacy, tolerability, and safety, including autoantibody generation, are lacking. 


\section{Conclusion}

Many patients do not achieve the recommended goals despite maximal statin therapy, and some patients cannot tolerate high-dose statin therapy. Available agents combined with statins can provide additional benefits on LDL-C reduction, and agents in development may increase therapeutic options. Drugs affecting old players in lipoprotein metabolism, such as apoB and MTP, have been recently approved for the management of dyslipidemia in FH patients (Table 1). Genetic insights into mechanisms underlying regulation of LDL-C levels have identified PCSK9 as a key target and led to the development of novel agents that are still undergoing testing to determine efficacy and safety (Table 1). Drugs increasing HDL may not be all alike and require adequate scrutiny of the mechanisms involved; increasing apoA-I may represent the best approach (Table 1). The most promising approaches, such as inhibitors of the synthesis of apoB containing lipoproteins or PCSK9 inhibitors, all decrease LDL as well. Until we have a better understanding of these issues, further LDL lowering in high-risk and very high-risk individuals represents the soundest clinical approach.

\section{Acknowledgments}

GDN is supported by grants from Fondazione Cariplo (2010-0768), Società Italiana Studio Aterosclerosi, Lombardia Chapter, PUR 2009, University of Milan.

\section{Disclosure}

The author reports no conflicts of interest in this work.

\section{References}

1. Norata GD, Tibolla G, Catapano AL. Gene silencing approaches for the management of dyslipidaemia. Trends Pharmacol Sci. 2013;34(4): 198-205.

2. Norata GD, Ballantyne CM, Catapano AL. New therapeutic principles in dyslipidaemia: Focus on LDL and Lp(a) lowering drugs. Eur Heart J. Epub March 18, 2013

3. Crooke ST. Progress in antisense technology. Annu Rev Med. 2004;55 61-95.

4. Yu RZ, Kim TW, Hong A, Watanabe TA, Gaus HJ, Geary RS. Cross-species pharmacokinetic comparison from mouse to man of a second-generation antisense oligonucleotide, ISIS 301012, targeting human apolipoprotein B-100. Drug Metab Dispos. 2007;35(3): 460-468.

5. Crooke RM, Graham MJ, Lemonidis KM, Whipple CP, Koo S, Perera RJ. An apolipoprotein B antisense oligonucleotide lowers LDL cholesterol in hyperlipidemic mice without causing hepatic steatosis. J Lipid Res. 2005;46(5):872-884

6. Visser ME, Witztum JL, Stroes ES, Kastelein JJ. Antisense oligonucleotides for the treatment of dyslipidaemia. Eur Heart J. 2012; 33(12): 1451-1458.

7. Akdim F, Tribble DL, Flaim JD, et al. Efficacy of apolipoprotein B synthesis inhibition in subjects with mild-to-moderate hyperlipidaemia. Eur Heart J. 2011;32(21):2650-2659.
8. Visser ME, Wagener G, Baker BF, et al. Mipomersen, an apolipoprotein B synthesis inhibitor, lowers low-density lipoprotein cholesterol in high-risk statin-intolerant patients: a randomized, double-blind, placebo-controlled trial. Eur Heart J. 2012;33(9):1142-1149.

9. Calandra S, Tarugi P, Speedy HE, Dean AF, Bertolini S, Shoulders CC. Mechanisms and genetic determinants regulating sterol absorption, circulating LDL levels, and sterol elimination: implications for classification and disease risk. J Lipid Res. 2011;52(11): 1885-1926.

10. Cuchel M, Bloedon LT, Szapary PO, et al. Inhibition of microsomal triglyceride transfer protein in familial hypercholesterolemia. $N \mathrm{Engl}$ J Med. 2007;356(2):148-156.

11. Samaha FF, McKenney J, Bloedon LT, Sasiela WJ, Rader DJ. Inhibition of microsomal triglyceride transfer protein alone or with ezetimibe in patients with moderate hypercholesterolemia. Nat Clin Pract Cardiovasc Med. 2008;5(8):497-505.

12. Dubuc G, Chamberland A, Wassef H, et al. Statins upregulate PCSK9, the gene encoding the proprotein convertase neural apoptosis-regulated convertase-1 implicated in familial hypercholesterolemia. Arterioscler Thromb Vasc Biol. 2004;24(8):1454-1459.

13. Li J, Tumanut C, Gavigan JA, et al. Secreted PCSK9 promotes LDL receptor degradation independently of proteolytic activity. Biochem J. 2007;406(2):203-207.

14. Tibolla G, Norata GD, Artali R, Meneghetti F, Catapano AL. Proprotein convertase subtilisin/kexin type 9 (PCSK9): from structure-function relation to therapeutic inhibition. Nutr Metab Cardiovasc Dis. 2011; 21(11):835-843.

15. Sun H, Samarghandi A, Zhang N, Yao Z, Xiong M, Teng BB. Proprotein convertase subtilisin/kexin type 9 interacts with apolipoprotein B and prevents its intracellular degradation, irrespective of the lowdensity lipoprotein receptor. Arterioscler Thromb Vasc Biol. 2012; 32(7):1585-1595.

16. Stein EA, Mellis S, Yancopoulos GD, et al. Effect of a monoclonal antibody to PCSK9 on LDL cholesterol. N Engl J Med. 2012 ; 366(12):1108-1118.

17. Dias C, Shaywitz A, Cooke B, et al. Effects of AMG 145, a fully human monoclonal antibody against pcsk9, on low-density lipoprotein cholesterol in subjects taking statins: a phase 1, randomized, doubleblind, placebo-controlled, ascending study. J Am Coll Cardiol. 2012; 59(Suppl 13):E1379.

18. McKenney JM, Koren MJ, Kereiakes DJ, Hanotin C, Ferrand AC, Stein EA. Safety and efficacy of a monoclonal antibody to proprotein convertase subtilisin/kexin type 9 serine protease, SAR236553/ REGN727, in patients with primary hypercholesterolemia receiving ongoing stable atorvastatin therapy. J Am Coll Cardiol. 2012;59(25): 2344-2353.

19. Stein EA, Gipe D, Bergeron J, et al. Effect of a monoclonal antibody to PCSK9, REGN727/SAR236553, to reduce low-density lipoprotein cholesterol in patients with heterozygous familial hypercholesterolaemia on stable statin dose with or without ezetimibe therapy: a phase 2 randomized controlled trial. Lancet. 2012;380(9836):29-36.

20. Alnylam Pharmaceuticals. Trial to evaluate safety and tolerability of ALN-PCS02 in subjects with elevated LDL-cholesterol (LDL-C). In: ClinicalTrials.gov [website on the Internet]. Bethesda, MD: US National Library of Medicine; 2011 [updated October 11,2012]. Available from: http:/www.clinicaltrials.gov/ct2/show/NCT01437059. NLM identifier: NCT01437059. Accessed May 1, 2013.

21. Cannon CP, Shah S, Dansky HM, et al. Safety of anacetrapib in patients with or at high risk for coronary heart disease. N Engl J Med. 2010; 363(25):2406-2415

22. Davidson M, Liu SX, Barter P, et al. Measurement of LDL-C after treatment with the CETP inhibitor anacetrapib. J Lipid Res. 2013; 54(2):467-472.

23. Nicholls SJ, Brewer HB, Kastelein JJ, et al. Effects of the CETP inhibitor evacetrapib administered as monotherapy or in combination with statins on HDL and LDL cholesterol: a randomized controlled trial. JAMA. 2011;306(19):2099-2109. 
24. von Eckardstein A, Nofer JR, Assmann G. High-density lipoproteins and arteriosclerosis. Role of cholesterol efflux and reverse cholesterol transport. Arterioscler Thromb Vasc Biol. 2001;21(1):13-27.

25. Khera AV, Cuchel M, de la Llera-Moya M, et al. Cholesterol efflux capacity, high-density lipoprotein function, and atherosclerosis. $N \mathrm{Engl}$ J Med. 2011;364(2):127-135.

26. Norata GD, Pirillo A, Ammirati E, Catapano AL. Emerging role of highdensity lipoproteins as a player in the immune system. Atherosclerosis. 2012;220(1):11-21.

27. Norata GD, Pirillo A, Catapano AL. HDLs, immunity, and atherosclerosis. Curr Opin Lipidol. 2011;22(5):410-416.

28. Norata GD, Björk H, Hamsten A, Catapano AL, Eriksson P. High-density lipoprotein subfraction 3 decreases ADAMTS-1 expression induced by lipopolysaccharide and tumor necrosis factor-alpha in human endothelial cells. Matrix Biol. 2004;22(7):557-560.

29. Pellegatta F, Bragheri M, Grigore L, et al. In vitro isolation of circulating endothelial progenitor cells is related to the high-density lipoprotein plasma levels. Int J Mol Med. 2006;17(2):203-208.

30. Norata GD, Pellegatta F, Hamsten A, Catapano AL, Eriksson P. Effects of HDL3 on the expression of matrix-degrading proteases in human endothelial cells. Int J Mol Med. 2003;12(1):73-78.

31. Voight BF, Peloso GM, Orho-Melander M, et al. Plasma HDL cholesterol and risk of myocardial infarction: a mendelian randomization study. Lancet. 2012;380(9841):572-580.

32. Lewis GF, Rader DJ. New insights into the regulation of HDL metabolism and reverse cholesterol transport. Circ Res. 2005;96(12):1221-1232.

33. Parini P, Rudel LL. Is there a need for cholesteryl ester transfer protein inhibition? Arterioscler Thromb Vasc Biol. 2003;23(3):374-375.

34. Barter PJ, Caulfield M, Eriksson M, et al. Effects of torcetrapib in patients at high risk for coronary events. $N$ Engl J Med. 2007;357(21): 2109-2122.

35. Rader DJ. Illuminating HDL - is it still a viable therapeutic target? N Engl J Med. 2007;357(21):2180-2183.

36. Forrest MJ, Bloomfield D, Briscoe RJ, et al. Torcetrapib-induced blood pressure elevation is independent of CETP inhibition and is accompanied by increased circulating levels of aldosterone. $\mathrm{Br} \mathrm{J}$ Pharmacol. 2008;154(7):1465-1473.

37. Hoffmann-La Roche Ltd. Roche provides update on Phase III study of dalcetrapib [press release] .Basel, Switzerland. May 7, 2012. Available from: http://www.roche.com/media/media_releases/medcor-2012-2005-07.htm. Accessed May 1, 2013.
38. Lüscher TF, Taddei S, Kaski JC, et al. Vascular effects and safety of dalcetrapib in patients with or at risk of coronary heart disease: The dal-VESSEL randomized clinical trial. Eur Heart J. 2012;33(7): 857-865.

39. Fayad ZA, Mani V, Woodward M, et al. Safety and efficacy of dalcetrapib on atherosclerotic disease using novel noninvasive multimodality imaging (dal-PLAQUE): a randomized clinical trial. Lancet. 2011;378(9802):1547-1559.

40. Lamon-Fava S, Diffenderfer MR, Barrett PH, et al. Extended-release niacin alters the metabolism of plasma apolipoprotein (Apo) A-I and ApoB-containing lipoproteins. Arterioscler Thromb Vasc Biol. 2008; 28(9):1672-1678.

41. Nicholls SJ, Gordon A, Johansson J, et al. Efficacy and safety of a novel oral inducer of apolipoprotein A-I synthesis in statin-treated patients with stable coronary artery disease a randomized controlled trial. J Am Coll Cardiol. 2011;57(9):1111-1119.

42. Tardif JC, Grégoire J, L'Allier PL, et al. Effects of reconstituted highdensity lipoprotein infusions on coronary atherosclerosis: a randomized controlled trial. JAMA. 2007;297(15):1675-1682.

43. Nissen SE, Tsunoda T, Tuzcu EM, et al. Effect of recombinant ApoA-I Milano on coronary atherosclerosis in patients with acute coronary syndromes: a randomized controlled trial. JAMA. 2003; 290(17):2292-2300.

44. Cerenis Therapeuitcs, SA. Effect of CER-001 on Atherosclerosis in Acute Coronary Syndrome (ACS) Patients - Efficacy and Safety: The CHI SQUARE Trial. In: ClinicalTrials.gov [website on the Internet]. Bethesda, MD: US National Library of Medicine; 2010. [updated October 4, 2012]. Available from: http://clinicaltrials.gov/ ct2/show/NCT01201837. NLM identifier: NCT01201837. Accessed May 1, 2013.

45. Norata GD, Sala F, Catapano AL, Fernandez-Hernando C. MicroRNAs and lipoproteins: a connection beyond atherosclerosis? Atherosclerosis. 2013;227(2):209-215.

46. D’Souza W, Stonik JA, Murphy A, et al. Structure/function relationships of apolipoprotein A-I mimetic peptides: implications for antiatherogenic activities of high-density lipoprotein. Circ Res. 2010;107(2): $217-227$.
Research Reports in Clinical Cardiology

\section{Publish your work in this journal}

Research Reports in Clinical Cardiology is an international, peerreviewed, open access journal publishing original research, reports, editorials, reviews and commentaries on all areas of cardiology in the clinic and laboratory. The manuscript management system is completely online and includes a very quick and fair peer-review system.

\section{Dovepress}

Visit http://www.dovepress.com/testimonials.php to read real quotes from published authors. 